\title{
New methods for space debris collision assessment
}

\author{
Daniel Casanova $^{1}$, Chiara Tardioli ${ }^{2}$ and Anne Lemaître ${ }^{1}$ \\ ${ }^{1}$ University of Namur, naXys - Department of Mathematics \\ 8 Rempart de la Vierge, 5000, Namur, Belgium \\ email: daniel.casanova@unamur. be \\ ${ }^{2}$ University of Strathclyde, Department of Mechanical \& Aerospace Engineering \\ 75 Montrose Street, Glasgow, UK
}

\begin{abstract}
Collisions between two pieces of space debris or between a piece of debris and an operative satellite is a real problem. Furthermore, collisions are responsible for the creation of new space debris systematically. The way to exclude the possibility of a collision consists of analysing the trajectories and looking for a time of coincidence. However, the analysis of all pairs of objects collected in a large orbit catalogue is unfeasible. The proposed method consists of reducing the possible pairs of candidates for a collision into a short list of pairs at real risk of collision. The method is based on a three-filter sequence: the first two filters are based on the geometry of the orbits, while the third one searches for a time of coincidence. This new method is tested resulting into an efficient tool for space debris collision assessment.
\end{abstract}

Keywords. Celestial Mechanics, Earth, ephemerides

\section{Introduction}

Since the launch of the first satellite Sputnik I in October 1957, more than 7,125 spacecraft have been launched into space. In addition to this population of satellites, we should include debris generated by the uncontrolled collision between Cosmos 2251 and Iridium 33, or by the intentional explosion of the Fengyun 1C. These events increased the population of space debris of about $40 \%$ in just two years (Pardini \& Anselmo (2011)). According to NASA, more than 21,000 pieces of space debris larger than $10 \mathrm{~cm}$ have been tracked and catalogued.

In this work, the initial conditions of space debris trajectories are determined by two different techniques. The first one uses a new orbit determination method based on the first integrals of the Kepler problem (Gronchi, Dimare \& Milani (2010)). The method produces a catalogue of fictitious orbits (Dimare et al. (2011)). The second method uses the Two Line Element (TLE) catalogue, which provides information of about 21,000 objects, grouping together active satellites and space debris. In particular, this work considers the TLE of the pieces of space debris generated by the previously mentioned catastrophic events.

Space debris collisions are a real problem. Space debris may cause serious damages to operative satellites and they have potentially devastating repercussions for communications and security. The goal of this work is to present and test an effective and realistic tool for the detection of all possible collisions between couples of objects in a large orbit catalogue. The method has been introduced in Casanova, Tardioli \& Lemaitre (2014) and consists of applying a sequence of three filters to significantly reduce the number of pairs candidates for a collision. Indeed, an all-to-all approach is unfeasible, since the computation of all possible pairs and the search for possible collisions between them 
has computational complexity of order $N^{2}$, where $N$ is the number of objects in the catalogue.

In this work, we compute the ephemerides of each piece of space debris using recent models developed by members of the Namur Center for Complex Systems (naXys): Valk, Lemaitre \& Anselmo (2008), Lemaitre, Delsate \& Valk (2009), Valk, Lemaitre \& Deleflie (2009), Valk et al. (2009), Delsate et al. (2010), Hubaux et al. (2012), Casanova \& Lemaitre (2014). The ephemerides are stored using direct access files.

The work is organized as follows: Section 2 briefly describes the ephemeris interpolation table. Section 3 focuses on the three-filter sequence method to assess space debris collisions. Afterwards, in Section 4 numerical experiments show the reliability of the filter sequence, and finally, we present the conclusions.

\section{Ephemeris interpolation table}

The evolution of each space debris object is computed numerically by a symplectic integration scheme (Hubaux et al. (2012)), which includes the Earth's gravitational potential, the luni-solar and planetary gravitational perturbations, and the direct solar radiation pressure. We propagate each object with a fixed time step, and we store all the ephemeris information in a direct access file (see Casanova, Tardioli \& Lemaitre (2014) for a detailed explanation). This technique allows us to compute the ephemerides of any object at any instant of time by a linear interpolation.

\section{Three-filter sequence}

The original three-filter sequence was established by Hoots, Crawford \& Roehrich (1984) to determine future close approaches between satellites. The method proposed in Casanova, Tardioli \& Lemaitre (2014) improves the previous one by using the ephemeris table instead of the osculating orbital elements, but also by applying some novel techniques. In the following we summarize the main features of the new three-filter sequence.

\subsection{Filter $I$}

Thanks to the ephemeris interpolation table, for each piece of space debris, it is possible to compute its geocentric distance $\vec{r}(t)$ and its time derivative $\dot{\vec{r}}(t)$ at any time $t$. We are interested in computing the maximum and minimum of this distance, which is equivalent to find the values where the derivative function $\dot{\vec{r}}(t)$ is equal to zero. This problem is solved by using a 'regula-falsi' algorithm similar to the one used in Milani et al. (2005). So, given a pair of objects of index $s_{1}, s_{2}$, we compute the maximum and minimum values of their geocentric distances, named $\max \left(r_{s_{j}}\right)$ and $\min \left(r_{s_{j}}\right)$, for $j=1,2$. Then, we set $q:=\max \left\{\min \left(r_{s_{1}}\right), \min \left(r_{s_{2}}\right)\right\}$ and $Q:=\min \left\{\max \left(r_{s_{1}}\right), \max \left(r_{s_{2}}\right)\right\}$. If the condition $|q-Q|>D$, with $D$ a fixed threshold distance, holds, then there is no possibility for an orbit crossing and the selected couple is rejected. Filter I improves the one presented by Hoots since a smaller number of false positives pass to the second filter.

\subsection{Filter II}

This filter is based on the definition of the orbit distance map introduced by Gronchi \& Tommei (2007). For each pair of objects it is possible to compute their orbit distance with sign at any instant of time of the interpolation process. If there is a change of sign in this distance function, an orbit crossing occurs. Hoots' second filter ensures that the Minimum Orbit Intersection Distance (MOID) is close to the line of nodes. However, the minimal points can be far away from the mutual nodes, especially for low mutual 
inclinations (Gronchi (2002)). Contrary, the orbit distance with sign is independent of the mutual nodes and it is proven to be more regular than the MOID in a neighbourhood of most crossing configurations.

\subsection{Filter III}

Filter III, also named time filter, computes the position of the two pieces of space debris looking for a time of coincidence. If there is a time where the distance between the two pieces is smaller than a threshold distance $D$, the pair is considered at real risk of collision, otherwise the possibility of a collision for the pair under consideration is excluded.

\section{Numerical experiments}

The effectiveness and reliability of the three-filter sequence has been tested through different populations of space debris. The procedure is the following. First, we compute the orbital evolution of all objects during 1 day, with a time span of $15 \mathrm{~min}$. Then, we create a direct access file with the ephemerides of all the objects at all time nodes (ephemeris interpolation table). Afterwards, we apply the three-filter sequence considering an interpolation time step of $7.5 \mathrm{~min}$ and a threshold distance of $2 \mathrm{~km}$.

The first space debris population consists of 864 objects in low-Earth orbit (LEO) region, i.e. with semimajor axis less than or equal to $8,600 \mathrm{~km}$. The initial orbital conditions have been determined by a new orbit determination method based on the first integrals of the Kepler problem (Gronchi, Dimare \& Milani (2010)). Table 1 illustrates the number of objects that have been rejected by each filter, concluding that there are no pairs at real risk of collision.

Table 1. 864 pieces of space debris. Ratio $A / m=0.01$.

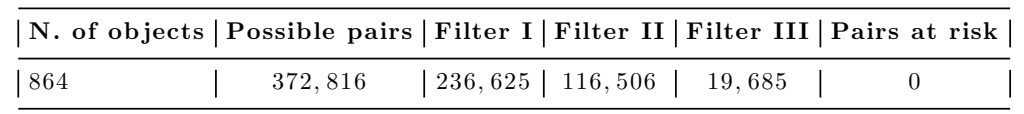

The second population is composed of 5,000 pieces of space debris, whose initial conditions have been obtained from the previous set of 864 objects by slightly modifying their inclinations. Table 2 shows the results of the filter sequence, concluding that 2, 461 pairs of objects are at real risk of collision.

Table 2. 5, 000 fictitious pieces of space debris. Ratio $A / m=0.01$

\begin{tabular}{|c|c|c|c|c|}
\hline N. of object & Possible pairs & Filter I | Filter II & Filter III & Pairs \\
\hline 5,0 & $12,497,500$ & 579 & 666,833 & 2,461 \\
\hline
\end{tabular}

An innovative part of this paper is that the three-filter sequence can be applied to a set of initial conditions taken from the Two Line Elements (TLE) catalogue. In particular, we consider the catastrophic events already mentioned in the introduction; the uncontrolled collision between Cosmos 2251 and Iridium 33, and the intentional explosion of the Fengyun 1C. These two events generated in total 4, 194 pieces of space debris. We consider that these objects have an area-to-mass ratio equal to $0.01 \mathrm{~m}^{2} / \mathrm{Kg}$. After applying the filter sequence to the population, we conclude that there are no pairs at real risk of collision, as we illustrate in Table 3.

Finally, we consider the same population of 4,194 pieces of space debris, but we randomize the ratio $A / m$ of each object in the range $[0.01,10]$. The results are shown in 
Table 3. 4, 194 pieces of space debris. Ratio $A / m=0.01$

\begin{tabular}{l|c|c|c|c|c|}
\hline N. of objects $\mid$ Possible pairs & Filter I & Filter II $\mid$ Filter III $\mid$ Pairs at risk | \\
\hline 4,194 & $8,792,721$ & $\mid 4,875,138$ & $3,194,869 \mid$ & 722,714 & 0 \\
\hline
\end{tabular}

Table 4 and they are quite similar to the previous population, concluding that there are no pairs at real risk of collision.

Table 4. 4, 194 pieces of space debris. Random ratio $A / m$

\begin{tabular}{|l|c|c|c|c|c|}
\hline N. of objects & Possible pairs & Filter I & Filter II & Filter III $\mid$ Pairs at risk | \\
\hline 4,194 & $8,792,721$ & $\mid 4,875,176$ & $3,194,784$ & 722,761 & 0 \\
\hline
\end{tabular}

\section{Conclusions}

This work proves the effectiveness and reliability of the three-filter sequence through four different experiments. The proposed sequence is a powerful method to exclude pairs of objects that are not at real risk of collision. At the same time, the pairs at real risk of collision are identified for possible collision avoidance analysis. Moreover, this filter sequence can be applied to any population of space debris whose initial conditions are obtained from the TLE catalogue. Future works will take into account the orbit uncertainty, in order to increase the reliability of the results.

\section{Acknowledgements}

This paper presents research results of the Belgian Network DYSCO (Dynamical Systems, Control, and Optimization), funded by the Interuniversity Attraction Poles Programme, initiated by the Belgian State, Science Policy Office. The scientific responsibility rests with its author(s).

\section{References}

Casanova, D., Tardioli, C., \& Lemaitre, A. 2014, MNRAS, 442 (4), 3235-3242

Casanova, D. \& Lemaitre, A. 2014, Submitted to Celest. Mech. Dyn. Astr.

Delsate, N., Lemaitre, A., Carletti, T., \& Robutel, P. 2010, Celest. Mech. Dyn. Astr., 108 (3), $275-300$

Dimare, L., Farnocchia, D., Gronchi, G. F., Milani, A., Bernardi, F., \& Rossi, A. 2011, Advanced Maui Optical and Space Surveillance Technologies Conference, 1, 51

Gronchi, G. F. 2002, SIAM J. Sci. Comput., 24 (1), 61-80

Gronchi, G. \& Tommei, G. 2007, Discret. Contin. Dyn-B., 7 (4), 755-778

Gronchi, G. F., Dimare, L., \& Milani, A. 2010, Celest. Mech. Dyn. Astr., 107 (3), 299-318

Hoots, F. R. \& Crawford, L. L., Roehrich, R. L. 1984, Celest. Mech., 33 (2), 143-158

Hubaux, C., Lemaitre, A., Delsate, N., \& Carletti, T. 2012, Adv. Sp. Res., 49 (10), 1472-1486

Lemaitre, A., Delsate, N., \& Valk, S. 2009, Celest. Mech. Dyn. Astr., 104 (4), 383-402

Milani, A., Tommei, G., Chesley, S., Sansaturio, M., \& Valsecchi, G. 2005, Icarus, 173 (2), $362-384$

Pardini, C. \& Anselmo, L. 2011, Adv. Sp. Res., 48 (3), 557-569

Valk, S., Lemaitre, A., \& Anselmo, L. 2008, Adv. Sp. Res., 41 (7), 1077-1090

Valk, S., Lemaitre, A., \& Deleflie, F. 2009, Adv. Sp. Res., 43 (7), 1070-1082

Valk, S., Delsate, N., Lemaitre, A., \& Carletti, T. 2009, Adv. Sp. Res., 43 (10), 1509-1526 